\title{
Asociación de factores de riesgo independientes con fracaso posextubación en pacientes desconectados de la ventilación
} mecánica

\author{
Martín Santibañez-Velázquez, ${ }^{*}$ Gabriela Medina-García ${ }^{1}$ y María E. Ocharán-Hernández² \\ ${ }^{1}$ Instituto Mexicano del Seguro Social, Centro Médico Nacional La Raza, Hospital de Especialidades; ${ }^{2}$ Instituto Politécnico Nacional, Escuela Superior \\ de Medicina, Coordinación de Investigación. Ciudad de México, México
}

\section{Resumen}

Introducción: Aun con adecuado protocolo de desconexión de la ventilación mecánica (DVM), el procedimiento falla en 15 a 30 \% de los casos. Objetivo: Evaluar la asociación entre factores de riesgo independientes y fracaso posextubación en pacientes con DVM en una unidad de cuidados intensivos. Método: Estudio de cohorte, Iongitudinal, prospectivo, analítico, que incluyó pacientes sometidos a ventilación mecánica por más de 24 horas y que fueron extubados. Se obtuvieron reportes preextubación de hemoglobina, albúmina, fósforo, índice cintura-cadera y puntuación SOFA. Se definió como fracaso de extubación al reinicio de la ventilación mecánica en 48 horas o menos. Resultados: Se extubaron 123 pacientes, 74 hombres (60\%); la edad promedio fue de $50 \pm 18$ años. Ocurrió fracaso de extubación en 37 (30 \%). Como factores de riesgo independentes se asoció hipoalbuminemia en 29 (23.8 \%, RR = 1.43, IC $95 \%=1.11-1.85)$ e hipofosfatemia en 18 $(14.6 \%, R R=2.98, I C 95 \%=1.66-5.35)$; se observaron dos o más factores de riesgo independientes en $22.7 \%$ (RR = 1.51 , IC $95 \%=1.14-2.00)$. Conclusiones: Identificar los factores de riesgo independentes antes de la DVM puede ayudar a reducir el fracaso de la extubación y la morbimortalidad asociada.

PALABRAS CLAVE: Desconexión. Ventilación mecánica. Fracaso de extubación. Factores de riesgo independientes.

\section{Association of independent risk factors with post-extubation failure in patients undergoing mechanical ventilation weaning}

\begin{abstract}
Introduction: Even with an adequate mechanical ventilation weaning (MVW) protocol, the procedure fails in 15 to $30 \%$ of cases. Objective: To assess the association between independent risk factors (IRFs) and post-extubation failure in patients undergoing MVW in an intensive care unit. Method: Longitudinal, prospective, analytical cohort study in patients on mechanical ventilation for more than 24 hours and who were extubated. Pre-extubation reports of hemoglobin, albumin, phosphorus, waist-hip ratio and SOFA score were obtained. Extubation failure was defined as resumption of mechanical ventilation within 48 hours or less. Results: 123 patients were extubated, out of whom 74 were males (60\%); average age was $50 \pm 18$ years. Extubation failure occurred in $37(30 \%)$. Hypoalbuminemia was associated as an independent risk factor in $29(23.8 \%$, $R R=1.43,95 \% \mathrm{Cl}=1.11-1.85)$ and hypophosphatemia was in $18(14.6 \%, R R=2.98,95 \% \mathrm{Cl}=1.66-5.35)$; two or more IRFs were observed in $22.7 \%(R R=1.51,95 \% \mathrm{Cl}=1.14-2.00)$. Conclusions: Identifying independent risk factors prior to MVW can help reduce the risk of extubation failure and associated morbidity and mortality.
\end{abstract}

KEY WORDS: Weaning. Mechanical ventilation. Extubation failure. Independent risk factors.

Correspondencia:

*Martín Santibañez-Velázquez

E-mail: sanvemart2@gmail.com
Fecha de recepción: 21-08-2019

Fecha de aceptación: 30-01-2020

DOI: $10.24875 /$ GMM.20005479
Gac Med Mex. 2020;156:549-555

Disponible en PubMed

www.gacetamedicademexico.com

0016-3813/๑ 2020 Academia Nacional de Medicina de México, A.C. Publicado por Permanyer. Este es un artículo open access bajo la licencia CC BY-NC-ND (http://creativecommons.org/licenses/by-nc-nd/4.0/). 


\section{Introducción}

La ventilación mecánica (VM) se emplea con la finalidad de brindar apoyo ventilatorio a los pacientes que, por alguna patología o intoxicación por agentes biológicos, químicos o físicos, presentan insuficiencia respiratoria aguda con alteraciones severas en el intercambio de gases (disoxia) y no logran cubrir las demandas metabólicas de oxígeno, con peligro inminente de pérdida de la vida.,

El retiro de la VM continúa siendo un problema importante en las unidades de cuidados intensivos. ${ }^{3,4}$ El retraso innecesario en la descontinuación de la VM incrementa las complicaciones, la morbimortalidad y los costos hospitalarios. ${ }^{5,6}$ Aun con un adecuado protocolo de desconexión de la VM, el fracaso de la extubación (FE) persiste en 15 a $30 \%$ de los casos. ${ }^{7,8}$ En las guías europeas se incluye un valor de la presión arterial de oxígeno/fracción inspirada de oxígeno $\left(\mathrm{PaO}_{2} / \mathrm{FIO}_{2}\right) \geq 150 \mathrm{~mm} \mathrm{Hg}$ entre los criterios para valorar el retiro de la ventilación mecánica. ${ }^{9,10}$

EI FE se define como la necesidad de apoyo mecánico de la ventilación durante las primeras 48 horas posextubación; la extubación se califica como exitosa si se tolera por más de este tiempo.11,12

Los factores de riesgo relacionados con el FE han sido investigados y reportados en diferentes estudios; ${ }^{13,14}$ entre ellos se encuentra la obesidad, que restringe la movilidad de la caja torácica y modifica los volúmenes pulmonares durante la ventilación mecánica, con lo que incrementa tres veces el riesgo de FE. ${ }^{15-17}$

En México, en 2016 se reportó una prevalencia combinada de sobrepeso y obesidad de $72.5 \%$ en adultos de 20 años y más. ${ }^{18}$

Los pacientes de las unidades de cuidados intensivos cursan con anemia de diferentes grados; se ha reportado que aquellos con $<7.5 \mathrm{~g} / \mathrm{dL}$ de hemoglobina tienen dos veces más riesgo de $\mathrm{FE} .{ }^{19} \mathrm{De}$ igual forma, debido al incremento de la respuesta metabólica al trauma y la pérdida intestinal de proteínas, estos pacientes cursan con hipoalbuminemia, la cual se asocia tres veces más a ventilación mecánica prolongada. ${ }^{20} \mathrm{La}$ hipofosfatemia es un trastorno electrolítico frecuente en las unidades de cuidados intensivos y se asocia a debilidad muscular del diafragma y músculos intercostales, la cual ocasiona decremento del volumen corriente en la respiración espontánea e incrementa al doble el riesgo de FE. ${ }^{21,22}$
La presente investigación se realizó con el objetivo de conocer si la asociación de anemia, hipoalbuminemia, hipofosfatemia y obesidad visceral, considerados factores de riesgo independientes, incrementa el riesgo de FE en pacientes de unidades de cuidados intensivos a quienes se retira la ventilación mecánica, asociación que ha sido poco investigada.

\section{Método}

Se estudió una cohorte prospectiva y longitudinal de pacientes atendidos en la Unidad de Cuidados Intensivos del Hospital de Especialidades "Antonio Fraga Mouret”, Centro Médico Nacional La Raza, correspondiente al Instituto Mexicano del Seguro Social, entre el 1 de mayo de 2018 y el 1 de mayo de 2019. El proyecto fue autorizado por el comité local de investigación, con el registro 2018-3501-047.

Previa firma de carta de consentimiento informado por los pacientes o sus representantes legales, se incluyeron aquellos en protocolo de retiro de la VM que reunieron los siguientes criterios de inclusión: edad de 16 a 75 años, con VM por más de 24 horas y menos de 20 días en modalidad de presión de soporte, presión positiva continua de la vía aérea y ventilación mecánica sincronizada intermitente; con medición del índice cintura-cadera, reporte de hemoglobina, albúmina y fósforo previo a la extubación; pacientes quirúrgicos y no quirúrgicos; con evaluación secuencial de fallas orgánicas y SOFA (Sequential Organ Failure Assessment) $\leq 9$.

El retiro de la VM y la extubación del paciente fueron indicados por el médico tratante. Una vez que el paciente fue extubado se registró la gasometría arterial preextubación, así como los niveles de albúmina, hemoglobina y fósforo y se observó la evolución del paciente durante las primeras 12, 24 y 48 horas. La tolerancia o no de la extubación fue valorada por el médico tratante en turno, quien decidió si el paciente era reintubado o no.

Se registró extubación exitosa si el paciente la toleraba por más de 48 horas y FE si el paciente fue reintubado en un periodo $\leq 48$ horas. Se registró la causa de la reintubación, la realización de traqueostomía, el tiempo de estancia y el motivo del egreso.

Al final se analizó la asociación del desenlace de la extubación con los factores de riesgo independientes. En la Figura 1 se muestra el diseño de la investigación. 


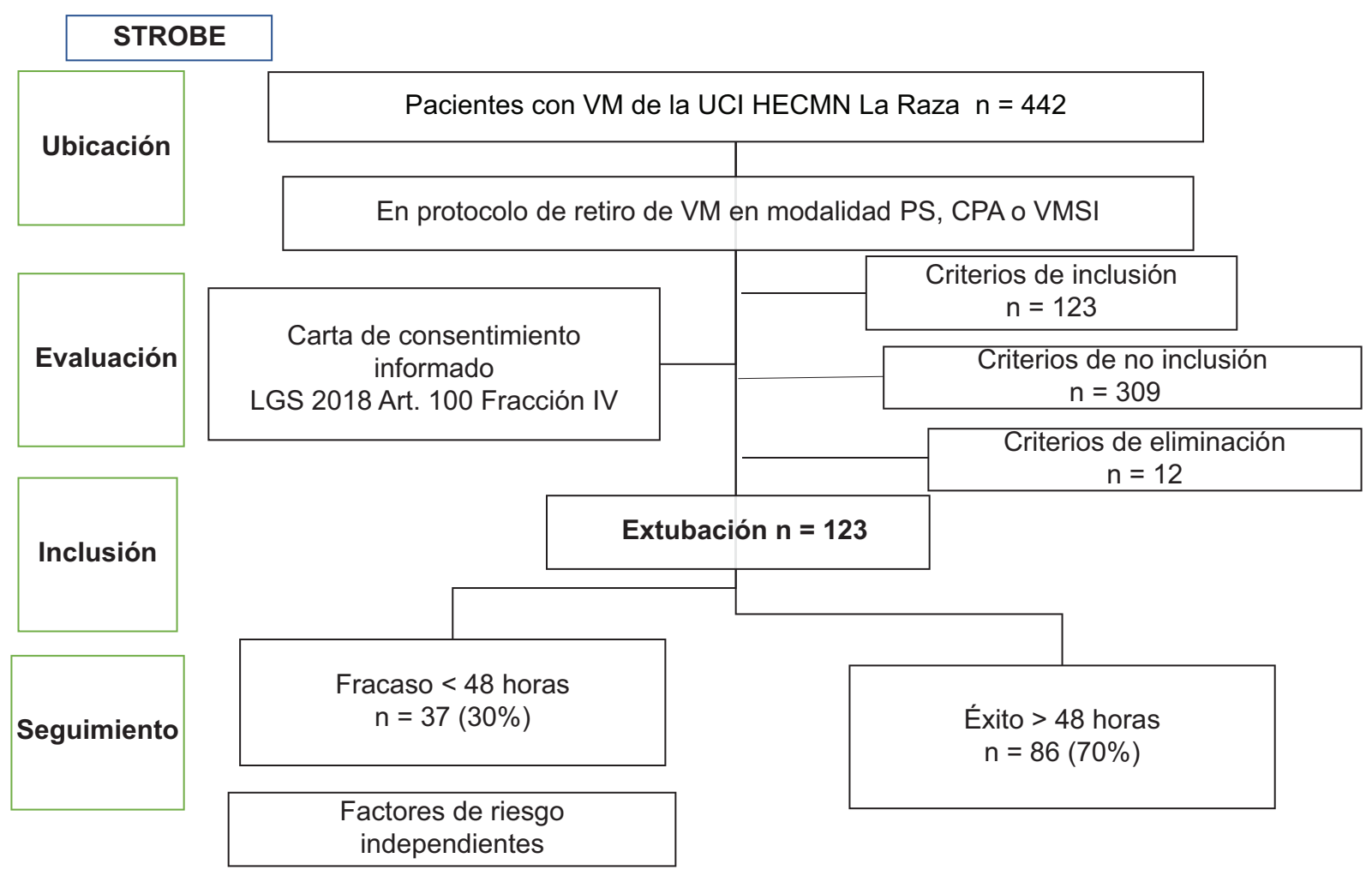

Figura 1. Algoritmo del desarrollo de la investigación. VM = ventilacion mecánica, UCI HECMN La Raza= Unidad de Cuidados Intensivos del Hospital de Especialidades, Centro Médico Nacional La Raza. $P S=$ presión de soporte, $C P A=$ presión positiva continua, VMSI = ventilación mecánica sincronizada intermitente.

Se calcularon frecuencias y porcentajes de las variables categóricas y se llevó a cabo análisis bivariado con la prueba de $\chi^{2}$ de Pearson $p<0.05$.

Se determinó media, desviación estándar e intervalos de confianza de $95 \%$ (IC $95 \%$ ) de las variables cuantitativas con distribución normal; se obtuvo mediana y rangos intercuartílicos (RIC) de las variables de libre distribución. Finalmente, se realizó análisis de regresión logística múltiple y se calculó el exponencial de beta para conocer el riesgo de FE. ${ }^{23}$

\section{Resultados}

Se estudiaron 123 participantes con retiro de la ventilación mecánica y que reunieron los criterios de inclusión, 64 hombres (60\%) y 49 mujeres (40\%), con media de edad de $55 \pm 15.9$ años y $44 \pm 18.4$ años, respectivamente. En relación con las características antropométricas se encontró estatura promedio de $1.68 \pm 0.077 \mathrm{~cm}$ y $1.56 \pm 0.075 \mathrm{~cm}$, peso promedio de $80 \pm 15.0 \mathrm{~kg}$ y $73 \pm 14.7 \mathrm{~kg}$, índice cintura-cadera de $1.0 \pm 0.21$ y $0.87 \pm 0.17$, respectivamente para hombres y mujeres. Se encontró obesidad visceral en 33 hombres (26.8\%) y 28 mujeres (22.7\%).

En 74 pacientes (60\%) se registraron causas quirúrgicas de ingreso a la unidad de cuidados intensivos: sepsis abdominal en $23(19 \%)$, posoperatorio de cirugía de tórax en 14 (12\%), laparotomía exploradora en $13(11 \%)$ y posoperatorio de nefrectomía en cinco (4\%). En 49 paciente (40\%) se registraron causas no quirúrgicas: neumonía intrahospitalaria en 12 (10\%), síndrome coronario agudo en 10 (9\%), síndrome de insuficiencia respiratoria aguda en seis $(5 \%)$ y neumonía adquirida en la comunidad en seis (5\%).

Los pacientes tuvieron una puntuación SOFA media de $6 \pm 2$ a su ingreso a la unidad de cuidados intensivos; las causas de ingreso y los datos demográficos se muestran en la Tabla 1.

Se registraron los parámetros bioquímicos y gasométricos previos a la extubación (Tabla 2).

Se encontró que en 78 pacientes (63.4\%) el tiempo con VM osciló de uno a cinco días; en 33 (26.8\%), de seis a 11 días; en 12 (9.7\%), de 12 a 18 días. En cuanto a los modos de VM antes de la extubación, en 57 pacientes ( $46.3 \%$ ) se registró presión de soporte, 
Tabla 1. Características demográficas de $\mathbf{1 2 3}$ pacientes con retiro de la ventilación mecánica y causas de ingreso a la unidad de cuidados intensivos

\begin{tabular}{|c|c|c|c|c|}
\hline \multirow[t]{2}{*}{ Variable } & \multicolumn{2}{|c|}{ Hombre $(n=74,60 \%)$} & \multicolumn{2}{|c|}{ Mujer $(n=49,40 \%)$} \\
\hline & n & $\%$ & n & $\%$ \\
\hline $\begin{array}{l}\text { Edad } \\
>60 \text { años } \\
<60 \text { años }\end{array}$ & $\begin{array}{l}43 \\
31\end{array}$ & $\begin{array}{c}35 \\
25.2\end{array}$ & $\begin{array}{l}25 \\
24\end{array}$ & $\begin{array}{c}20 \\
19.5\end{array}$ \\
\hline Obesidad visceral & 33 & 26.8 & 28 & 22.8 \\
\hline Sedentarismo & 60 & 48.8 & 34 & 27.6 \\
\hline Tabaquismo & 48 & 39 & 11 & 8.9 \\
\hline Hipertensión arterial sistémica & 37 & 30 & 18 & 14.6 \\
\hline Diabetes tipo 2 & 25 & 20.3 & 14 & 11.4 \\
\hline Síndrome coronario agudo & 15 & 12.2 & 2 & 1.8 \\
\hline Insuficiencia renal crónica & 8 & 6.5 & 5 & 4.1 \\
\hline Enfermedad inmunológica & 3 & 2.4 & 5 & 4.1 \\
\hline Enfermedad pulmonar obstructiva crónica & 5 & 4.1 & 1 & 0.8 \\
\hline $\begin{array}{l}\text { Causas quirúrgicas } \\
\text { Sepsis abdominal } \\
\text { Posoperados de cirugía torácica } \\
\text { Laparotomía exploradora }\end{array}$ & $\begin{array}{c}44 \\
13 \\
8 \\
7\end{array}$ & $\begin{array}{c}36 \\
10.6 \\
6.5 \\
5.7\end{array}$ & $\begin{array}{c}30 \\
10 \\
6 \\
6\end{array}$ & $\begin{array}{l}25 \\
8.1 \\
4.9 \\
4.9\end{array}$ \\
\hline $\begin{array}{l}\text { Causas no quirúrgicas } \\
\text { Neumonía intrahospitalaria } \\
\text { Síndrome coronario agudo } \\
\text { SIRA }\end{array}$ & $\begin{array}{c}30 \\
8 \\
9 \\
3\end{array}$ & $\begin{array}{l}25 \\
6.5 \\
7.3 \\
2.4\end{array}$ & $\begin{array}{l}19 \\
4 \\
1 \\
3\end{array}$ & $\begin{array}{l}16 \\
3.3 \\
0.8 \\
2.4\end{array}$ \\
\hline
\end{tabular}

con FE en 18 (14.6\%), RR = 1.09, IC $95 \%=0.64-$ 1.87; en $55(44.7 \%)$ se registró presión positiva continua de la vía aérea, con FE en 16 (13\%), $R R=0.95$, IC $95 \%$ = 0.55-1.65; y ventilación mecánica intermitente sincronizada en 11 (8.9\%), con FE en tres (2.4\%), RR =0.89, IC $95 \%=0.32-2.45$. Durante el protocolo de retiro de la VM, 113 pacientes $(91 \%)$ se pasaron a tubo en $\mathrm{T}$, de los cuales $34(26.8 \%)$ presentaron FE (RR $=1.00$, IC $95 \%=0.89-1.12)$. No se indicó tubo en $\mathrm{T}$ a 10 pacientes, tres $(2.4 \%)$ de estos presentaron FE (RR =0.99, IC $95 \%=0.27-3.64)$. Los factores de riesgo de la población estudiada que presentó FE se muestran en la Tabla 3.

En la presente investigación, 37 pacientes fueron extubados con una relación $\mathrm{PaO}_{2} / \mathrm{FIO}_{2}<200$ (30\%), de los cuales $12 \%$ presentó FE.

Aun cuando se realizó adecuado protocolo para el retiro de la VM, ocurrió FE en 37 pacientes (30\%), sin diferencia significativa entre los sexos $(R R=1.11$, IC $95 \%=0.82-1.50, p=0.485$ ).

Se realizó análisis de regresión logística de los factores de riesgo independientes considerados en el
FE; los resultados se muestran en la Tabla 4. Los factores de riesgo independientes incluidos fueron hipoalbuminemia, hipofosfatemia y la asociación de dos o más factores de riesgo $(p=0.013)$ :

$$
y=-0.302+(0.913)+(1.33)+(0.788)
$$

La probabilidad más alta de FE fue de $37 \%$.

Las principales causas que justificaron la reintubación en la población estudiada durante las primeras 12 a 48 horas posextubación se muestran en la Figura 2.

De los pacientes que presentaron FE, 17 (14\%) egresaron sin VM, 11 (9\%) con VM, 10 con traqueostomía (8\%) y uno con VM por máximo beneficio. La mortalidad en estos pacientes fue de $8 \%$, con tiempo de estancia de siete días (RIC = 4-12).

Por mejoría egresaron 114 pacientes (92\%).

\section{Discusión}

En la cohorte analizada, los pacientes extubados previo protocolo de retiro de la VM no mostraron diferencias significativas en las condiciones basales. La 
Tabla 2. Características bioquímicas y gasométricas de la población previo a la extubación

\begin{tabular}{|c|c|c|}
\hline Variable & Hombre & Mujer \\
\hline Hemoglobina, 8 a 10 g/dL* & $10.14 \pm 2.06$ & $10.14 \pm 1.69$ \\
\hline Albúmina $<3 \mathrm{~g} / \mathrm{dL}^{*}$ & $2.60 \pm 0.71$ & $2.75 \pm 0.92$ \\
\hline Fósforo $<2.5 \mathrm{~g} / \mathrm{dL}^{*}$ & $3.14 \pm 1.39$ & $2.91 \pm 1.05$ \\
\hline $\mathrm{FIO}_{2}(\%)$ & $42 \pm 9.4$ & $42 \pm 9.2$ \\
\hline PEEP $(\mathrm{mm} \mathrm{Hg})$ & $4.5 \pm 0.86$ & $4.8 \pm 0.76$ \\
\hline $\mathrm{pH}$ & $7.43 \pm 0.06$ & $7.43 \pm 0.06$ \\
\hline $\mathrm{PaO}_{2}(\mathrm{~mm} \mathrm{Hg})$ & $96.8 \pm 26.7$ & $98.6 \pm 34.7$ \\
\hline $\mathrm{PaCO}_{2}(\mathrm{~mm} \mathrm{Hg})$ & $33.9 \pm 6.33$ & $33.4 \pm 9.64$ \\
\hline $\mathrm{HCO}_{3}(\mathrm{~mm} \mathrm{Hg})$ & $23.3 \pm 5.15$ & $22.5 \pm 4.64$ \\
\hline Índice respiratorio & $1.12 \pm 0.75$ & $1.03 \pm 0.69$ \\
\hline Lactato (mg/dL) & $1.82 \pm 1.66$ & $1.64 \pm 0.62$ \\
\hline $\mathrm{PaO}_{2} / \mathrm{FIO}_{2}(\mathrm{~mm} \mathrm{Hg})$ & $246 \pm 75.5$ & $239 \pm 63.7$ \\
\hline Tiempo con VM (días) & $4.5 \pm 3.6$ & $5.6 \pm 4.4$ \\
\hline \multicolumn{3}{|c|}{$\begin{array}{l}{ }^{*} \mathrm{~F} \text { Factores de riesgo independientes. } \\
\mathrm{FIO}_{2}=\text { fracción inspirada de oxígeno, } \mathrm{PEEP}=\text { presión positiva al final de la expiración, } \\
\mathrm{PaO}_{2}=\text { presión arterial de oxígeno, } \mathrm{PaCO}=\text { = presión arterial de dióxido de carbono, } \\
\mathrm{HCO}_{3}=\text { bicarbonato, } \mathrm{PaO}_{2} / \mathrm{FIO}_{2}=\text { relación presión arterial de oxígeno entre fracción } \\
\text { inspirada de oxígeno, } \mathrm{VM}=\text { ventilación mecánica. }\end{array}$} \\
\hline
\end{tabular}

hipoalbuminemia e hipofosfatemia fueron factores de riesgo independientes para FE y la asociación de dos o más factores de riesgo independientes incrementó $37 \%$ la probabilidad de FE, tanto en pacientes quirúrgicos como no quirúrgicos.

La hipoalbuminemia se presentó con una alta frecuencia y fue identificada como un factor de riesgo independiente para FE. La hipoalbuminemia es frecuente en los pacientes de las unidades de cuidados intensivos, es causada por diferentes factores y se asocia a mayor tiempo de estancia hospitalaria, mayor riesgo de FE y mayor mortalidad. Los resultados de esta investigación fortalecen los encontrados en otros estudios respecto a la asociación de la hipoalbuminemia y el mayor riesgo de FE. ${ }^{20}$

Se encontró que la hipofosfatemia es un trastorno electrolítico frecuente en los pacientes de las unidades de cuidados intensivos y resultó ser un factor de riesgo independiente para $\mathrm{FE}$, hallazgos semejantes a los reportados en la literatura internacional, en la que se refiere que la hipofosfatemia se asocia a mayor tiempo de estancia en la unidades de cuidados intensivo y mayor riesgo de FE. ${ }^{21,22}$

En nuestra investigación existió fuerte riesgo de FE al asociarse más de dos factores de riesgo independientes,

\section{Causas de fracaso al retiro de la ventilación mecánica}

Causa Frecuencia Porcentaje

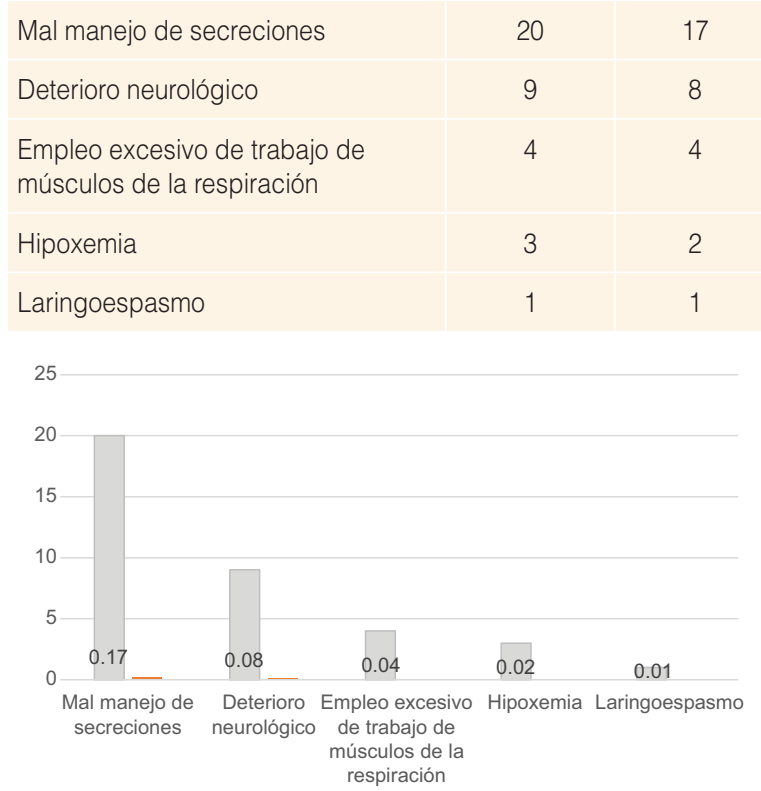

Figura 2. Eventos de reintubación y porcentaje agrupado por causas.

a diferencia de otros estudios en los cuales se analizaron estos factores de riesgo por separado.

En diferentes análisis se reporta que la hemoglobina $<7 \mathrm{~g} / \mathrm{dL}$ se asocia a riesgo alto de fracaso posextubación; en nuestra investigación no se encontró asociación entre la media de hemoglobina $10.14 \pm 2.06 \mathrm{~g} / \mathrm{dL}$ y el riesgo de $\mathrm{FE}$, lo cual concuerda con la recomendación en los criterios para extubación de que los niveles de hemoglobina sean de $\geq 8 \mathrm{~g} / \mathrm{dL}^{.19,20}$

La obesidad visceral en la presente investigación se observó con mayor frecuencia en los hombres, si bien no se identificó asociación del sexo con FE. En varias revisiones se alude a la asociación entre obesidad global y FE, sin embargo, existen pocos estudios en los cuales se reporte la asociación de obesidad visceral y riesgo de FE. ${ }^{16,17}$

Las comorbilidades como el sedentarismo, tabaquismo, diabetes tipo 2, hipertensión arterial sistémica, síndrome coronario agudo e insuficiencia renal crónica fueron más frecuentes en los hombres y las inmunológicas en las mujeres, de igual forma las causas quirúrgicas y no quirúrgicas de ingreso fueron más frecuentes en los hombre. No se encontró asociación entre la causa de ingreso y el riesgo de FE.

Respecto al modo de VM previo a la extubación, no se encontró diferencia significativa entre presión 
Tabla 3. Factores de riesgo en pacientes de la unidad de cuidados intensivos en quienes se retiró la ventilación mecánica

\begin{tabular}{|c|c|c|c|c|c|c|c|}
\hline \multirow[t]{2}{*}{ Variables } & \multicolumn{2}{|c|}{$F E<48$ horas $(n=37)$} & \multicolumn{2}{|c|}{ Extubación exitosa $(n=86)$} & \multirow[t]{2}{*}{ RR } & \multirow[t]{2}{*}{ IC $95 \%$} & \multirow[t]{2}{*}{ p } \\
\hline & $n$ & $\%$ & $n$ & $\%$ & & & \\
\hline Sexo masculino & 24 & 20 & 50 & 41 & 1.11 & $0.82-1.50$ & 0.485 \\
\hline Edad $>60$ años & 21 & 17 & 47 & 39 & 1.03 & $0.73-1.46$ & 0.829 \\
\hline Factores quirúrgicos & 20 & 17 & 54 & 44 & 1.28 & $0.75-2.19$ & 0.366 \\
\hline Obesidad visceral & 21 & 17 & 40 & 66 & 1.22 & $0.85-1.75$ & 0.297 \\
\hline Albúmina $<3 \mathrm{~g} / \mathrm{dL}^{*}$ & 29 & 24 & 47 & 39 & 1.43 & $1.11-1.85$ & 0.013 \\
\hline $\mathrm{Hb}, 8$ a $10 \mathrm{~g} / \mathrm{dL}$ & 18 & 15 & 42 & 34 & 1.00 & $0.46-2.17$ & 0.98 \\
\hline Fósforo $<2.5 \mathrm{mg} / \mathrm{dL}^{*}$ & 18 & 15 & 14 & 12 & 2.98 & $1.66-5.35$ & 0.000 \\
\hline Dos o más FRI* & 28 & 23 & 43 & 35 & 1.51 & $1.14-2.00$ & 0.008 \\
\hline Tiempo con VM, 6-11 días* & 15 & 12 & 13 & 10 & 1.85 & $1.10-3.13$ & 0.024 \\
\hline $\mathrm{PaO}_{2} / \mathrm{FIO}_{2}<200^{*}$ & 15 & 12 & 22 & 18 & 1.74 & $1.00-3.01$ & 0.051 \\
\hline Presión de soporte & 18 & 15 & 39 & 32 & 1.09 & $0.64-1.87$ & 0.113 \\
\hline CPAP & 16 & 13 & 39 & 32 & 0.95 & $0.55-1.65$ & 0.046 \\
\hline VMSI & 3 & 2 & 8 & 6.5 & 0.89 & $0.32-2.45$ & 0.045 \\
\hline
\end{tabular}

*Existe asociación.

$\mathrm{FE}=$ fracaso posextubación, $\mathrm{Hb}=$ hemoglobina, $\mathrm{VM}$ = ventilación mecánica, $\mathrm{PaO}_{2} / \mathrm{FIO}_{2}=$ relación presión arterial de oxígeno entre fracción inspirada de oxígeno preextubación, $\mathrm{FRI}=$ factores de riesgo independientes, $\mathrm{PS}=$ presión de soporte, $\mathrm{CPAP}=$ presión positiva continúa de la vía aérea, VMSI = ventilación mecánica sincronizada intermitente,

IC $95 \%$ = intervalo de confianza del $95 \%, \mathrm{RR}=$ riesgo relativo. $\mathrm{p}<0.05$.

Tabla 4. Factores de riesgo independientes en el modelo de regresión logística para fracaso posextubación en pacientes de una unidad de cuidados intensivos

\begin{tabular}{|c|c|c|c|c|}
\hline Variable & Beta & EE & RM & IC $95 \%$ \\
\hline Obesidad visceral & 0.048 & 0.444 & 1.049 & $0.85-1.75$ \\
\hline $\begin{array}{l}\text { Hemoglobina, } 8 \text { a } \\
10 \mathrm{~g} / \mathrm{dL}\end{array}$ & -0.794 & 0.586 & 0.452 & $0.46-2.17$ \\
\hline Albúmina $<3 \mathrm{~g} / \mathrm{dL}^{*}$ & 0.913 & 0.556 & 2.492 & $1.11-1.85$ \\
\hline Fósforo $<2.5 \mathrm{mg} / \mathrm{dL}^{*}$ & 1.337 & 0.510 & 3.807 & $1.66-5.35$ \\
\hline Dos o más FRI* & 0.788 & 0.723 & 2.200 & $1.14-2.00$ \\
\hline Constante & -0.302 & 0.482 & 0.739 & \\
\hline
\end{tabular}

*Factor de riesgo.

$\mathrm{FRI}=$ factores de riesgo independientes, $\mathrm{EE}=$ error estándar, $\mathrm{RM}=$ exponencial Beta, IC $95 \%$ = intervalo de confianza de $95 \%$.

de soporte, presión positiva continua de la vía aérea, ventilación mecánica intermitente sincronizada y FE. Existen pocos estudios de cohorte prospectivos que aludan al modo de la VM y su asociación con FE. ${ }^{3,4}$

En nuestro estudio observamos mayor frecuencia de $\mathrm{FE}$ en los pacientes con relación $\mathrm{PaO}_{2} /$ $\mathrm{FIO}_{2} \leq 200 \mathrm{~mm} \mathrm{Hg}$, por lo que diferimos con la recomendación de las guías europeas ${ }^{9,10}$ y sugerimos que no deben extubarse los pacientes con relación $\mathrm{PaO}_{2} /$ $\mathrm{FIO}_{2} \leq 200 \mathrm{~mm} \mathrm{Hg}$.

Las principales causas que justificaron la reintubación en la población estudiada fueron semejantes a las reportadas en la literatura. ${ }^{4,6} \mathrm{La}$ frecuencia de FE coincidió con la descrita en algunos estudios multicéntricos retrospectivos, en los cuales se reportó de 15 a $30 \%$. $^{6-8}$ Una vez identificados los factores de riesgo independientes consideramos que deben corregirse antes de llevar a cabo la extubación, con la finalidad de reducir el porcentaje de FE.

Al igual que lo reportado en otros estudios, el fracaso posextubación estuvo fuertemente asociado a incremento de la morbilidad y mortalidad. ${ }^{13,14}$

Las limitaciones del estudio consistieron en la variabilidad de criterios en el protocolo de extubación; derivado de ello, un gran número de sujetos fueron extubados con $\mathrm{PaO}_{2} / \mathrm{FlO}_{2}>150 \mathrm{~mm} \mathrm{Hg}$, pero $<200 \mathrm{~mm} \mathrm{Hg}$. Como consecuencia, el riesgo de FE fue mayor. Dado que el seguimiento de los sujetos de estudio terminó al concluir las 48 horas siguientes a la extubación, no fue posible conocer si posteriormente tuvieron que ser reintubados en piso.

Como fortalezas de nuestra investigación puede mencionarse que contribuye al conocimiento de la 
frecuencia de FE en nuestra población y algunos factores de riesgo independientes que lo favorecen, lo cual servirá como punto de partida para futuros estudios dirigidos a reducir el riesgo de FE.

\section{Conclusiones}

La presencia de hipoalbuminemia o hipofosfatemia incrementa el riesgo de FE en los pacientes de las unidades de cuidados intensivos que son extubados, riesgo que se incrementa al doble cuando se asocian dos o más factores de riesgo independientes. La identificación de estos factores de riesgo independientes antes de la extubación puede tener una potencial utilidad clínica para tomar la decisión en el proceso de extubación y reducir el riesgo de reintubación.

\section{Agradecimientos}

Con gran respeto se agradece a Juan $\mathrm{O}$. Talavera, Marcela Rodríguez Pérez, Rodolfo Rivas Ruiz, Elpidio Cruz Martínez y Luis Javier Jara Quezada, por la revisión del presente manuscrito. También, en forma especial, al Instituto Politécnico Nacional y al personal docente que labora ahí.

\section{Conflicto de Intereses}

Los autores declaramos no tener ningún conflicto de intereses.

\section{Financiamiento}

No se recibió ninguna subvención para este estudio.

\section{Responsabilidades éticas}

Protección de personas y animales. Los autores declaran que los procedimientos seguidos se apegaron a las normas éticas del comité de experimentación humana responsable y a los lineamientos de la Asociación Médica Mundial y la Declaración de Helsinki.

Confidencialidad de los datos. Los autores declaran que siguieron los protocolos de su centro de trabajo sobre la publicación de datos de pacientes.

Derecho a la privacidad y consentimiento informado. Se obtuvo por escrito el consentimiento informado de los pacientes, documento que obra en poder de los autores.

\section{Bibliografía}

1. Liu J, Shen F, Teboul J, Anguel N, Beurton A, Nadia Bezaz N, et al. How mechanical ventilation measurement, cutoff and duration affect rapid shallow breathing index accuracy: a randomized trial. J Clin Med Res. 2017;9:289-296. DOI: 10.14740/jocmr2856w

2. Pellegrini J, Moraes R, Maccari J, Oliveira R, Savi A, Ribeiro R, et al. Spontaneous breathing trials with T-piece or pressure support ventilation. Resp Care. 2016;61:1693-1703. DOI: 10.4187/respcare.04816

3. Cook D, Rocker G, Marshall J, Sjokvist P, Dodek P, Griffith L, et al. Withdrawal of mechanical ventilation in anticipation of death in the intensive care unit. N Eng J Med. 2003;349:1123-1132. DOI: 10.1056/NEJMoa030083

4. Gattinoni L, Marini JJ, Collino F, Maiolo G, Rapetti F, Tonetti T, et al. The future of mechanical ventilation: lessons from the present and the past. Crit Care. 2017;21:183. DOI: 10.1186/s13054-017-1750-x

5. Rojek-Jarmuła A, Hombach R, Krzych L. APACHE II score cannot predict successful weaning from prolonged mechanical ventilation. Chron Respir Dis. 2017;4:1-6. DOI: 10.1177/1479972316687100

6. Boles JM, Bion J, Connors A, Herridge M, Marsh B, Melote C, et al. Weaning from mechanical ventilation. Eur Respir J. 2007;29:1033-1056. DOI: 10.1183/09031936.00010206

7. Thille AW, Boissier F, Ghezala H, Razaz K, Mekontso-Dessap A, Brun-Buisson C, et al. Easily identified at-risk patients for extubation failure may benefit from noninvasive ventilation: a prospective before-after study. Crit Care Med. 2016;20:48. DOI: 10.1186/s13054-016-1228-2

8. Liu J, Shen F, Teboul JL, Anguel N, Beurton A, Bezaz N, et al. Cardiac dysfunction induced by weaning from mechanical ventilation: incidence, risk factors, and effects of fluid removal. Crit Care. 2016;20:1-14. DOI: 10.1186/s13054-016-1533-9

9. Macintyre NR, Cook DJ, Ely W, Epstein S, Fink JB, Heffner J, et al. Evidence-based guidelines for weaning and discontinuing ventilatory support: a collective task force facilitated by the American College of Chest Physicians; the American Association for Respiratory Care; and the American College of Critical Care Medicine. Chest. 2001;120:375S-395S. DOI: 10.1378/chest.120.6_suppl.375s

10. Shaikh $H$, Morales $D$, Franco $L$. Weaning from mechanical ventilation. Semin RespirCritCare Med. 2014;35:451-468. DOI: 10.1055/s-0034-1381953

11. Wu TJ, Shiao JS, Yu HL, Sheng Lai RS. An integrative index for predicting extubation outcomes after successful completion of a spontaneous breathing trial in an adult medical intensive care unit. $\mathrm{J}$ Intensive Care Med. 2019;34:640-645. DOI: 10.1177/0885066617706688

12. Piriyapatsom K, Williams E, Waak K, Ladha K, Eikermann M, Schmidt U, et al. Prospective observational study of predictors of re-intubation foIlowing extubation in the surgical ICU. Resp Care. 2016;61:306-315. DOI: 10.4187/respcare.04269

13. Thille W, Boissier F, Ghezala B, Razazi K, Mekontso-Dessap A, Brun-Buisson C. Risk factors for and prediction by caregivers of extubation failure in ICU patients: a prospective study. Crit Care Med. 2014;43:613-620. DOI: 10.1097/CCM.0000000000000748

14. Tischenkel BR, Gong MN, Shiloh AL, Pittignano VC, Keschner YG, Glueck JA, et al. Daytime versus nighttime extubations: a comparison of reintubation, length of stay, and mortality. J Intensive Care Med. 2016;31:118-126. DOI: 10.1177/0885066614531392

15. Kumar S, Doo K, Brammeier J, Lane Ch, Liebler J. Super obesity in the medical intensive care unit. J Intensive Care Med. 2020;35:478-484. DOI: $10.1177 / 0885066618761363$

16. Mahul M, Jung B, Galia F, Molinar N, Jong A, Coisel Y, et al. Spontaneous breathing trial and postextubation work of breathing in morbidly obese critically ill patients. Crit Care. 2016;20:1-12. DOI: 10.1186/ s13054-016-1457-4

17. Chao CM, Lai CC, Cheng AC, Chiang SR, Liu WL, Ho C, et al. Establishing failure predictors for the planned extubation of overweight and obese patients. Plos One. 2017;16:1-10. DOI: 10.1371/journal.pone.0183360

18. Encuesta Nacional de Salud y Nutrición de Medio Camino 2016. Resultados Nacionales. México: Instituto Nacional de Salud Pública; 2017.

19. Lai YC, Ruan SY, Huang CT, Kuo PH, Yu CJ. Hemoglobin levels and weaning outcome of mechanical ventilation in difficult-to-wean patients: a retrospective cohort study. PLoS One. 2013;8:1-6. DOI: 10.1371/journal.pone. 0073743

20. Liang G, Liu T, Zeng Y, Shi Y, Yang W, Yang Y. et al. Characteristics of subjects who failed a 120-minute spontaneous breathing trial. Respir Care. 2018;63:388-394. DOI: 10.4187/respcare.05820

21. Liu B, Cheng Y, Shen F, Wang Y, Wu Y, Yao L, et al. Hypophosphatemia is associated with poor prognosis of critically ill patients: a meta-analysis of 1555 patients. Zhonghua Wei Zhong Bing Ji Jiu Yi Xue. 2018;30:3440. DOI: 10.3760/cma.j.issn.2095-4352.2018.01.007

22. Zhao Y, Li Z, Shi Y, Cao G, Meng F, Zhu W, et al. Effect of hypophosphatemia on the withdrawal of mechanical ventilation in patients with acute exacerbations of chronic obstructive pulmonary disease. Biomed Rep. 2016;4:413-416. DOI: 10.3892/br.2016.605

23. Talavera OJ, Rivas RR. IV. Pertinencia de la prueba estadística. Rev Med Inst Mex Seguro Soc. 2013;51:S30-S34. 